\title{
Discovery of Relevant Response in Infected Potato Plants from Time Series of Gene Expression Data
}

\author{
Dragan Gamberger ${ }^{1} * \mathbb{E}$, Tjaša Stare ${ }^{2}$, Dragana Miljkovic ${ }^{3}$, Kristina Gruden ${ }^{4}$ and Nada Lavrač 5 \\ 1 Rudjer Bošković Institute, Bijenička cesta 54, 10000 Zagreb, Croatia \\ 2 National Institute of Biology, 1000 Ljubljana, Slovenia; tjasa.stare@nib.si \\ 3 Jožef Stefan Institute, 1000 Ljubljana, Slovenia; dragana.miljkovic@ijs.si \\ 4 National Institute of Biology, 1000 Ljubljana, Slovenia; Kristina.Gruden@nib.si \\ 5 Jožef Stefan Institute, Ljubljana, Slovenia, University of Nova Gorica, 5000 Nova Gorica, Slovenia; \\ Nada.Lavrac@ijs.si \\ * Correspondence: dragan.gamberger@irb.hr; Tel.: +385-1-456-1142
}

Received: 2 November 2018; Accepted: 8 January 2019; Published: 16 January 2019

\begin{abstract}
The paper presents a methodology for analyzing time series of gene expression data collected from the leaves of potato virus Y (PVY) infected and non-infected potato plants, with the aim to identify significant differences between the two sets of potato plants' characteristic for various time points. We aim at identifying differentially-expressed genes whose expression values are statistically significantly different in the set of PVY infected potato plants compared to non-infected plants, and which demonstrate also statistically significant changes of expression values of genes of PVY infected potato plants in time. The novelty of the approach includes stratified data randomization used in estimating the statistical properties of gene expression of the samples in the control set of non-infected potato plants. A novel estimate that computes the relative minimal distance between the samples has been defined that enables reliable identification of the differences between the target and control datasets when these sets are small. The relevance of the outcomes is demonstrated by visualizing the relative minimal distance of gene expression changes in time for three different types of potato leaves for the genes that have been identified as relevant by the proposed methodology.
\end{abstract}

Keywords: gene expression time series; potato virus infections; variance-stabilized data; randomization test; stratified randomization; relative minimal distance of samples

\section{Introduction}

Potato (Solanum tuberosum L.) is the most widely grown tuber crop in the world, and the fourth largest food crop in terms of fresh produce, after rice, wheat, and tomato. Potato virus Y (PVY) is a member of the Potyviridae family and, economically, it is one of the most important potato pathogens, with PVY ${ }^{\text {NTN }}$ being, worldwide, an aggressive isolate that induces severe symptoms in sensitive potato cultivars [1-3]. The interaction between a plant and its pathogen initiates a complex signaling network, resulting in massive changes of the gene activity and extensive reprogramming of the cell metabolism $[4,5]$.

Salicylic acid (SA) has shown to mediate resistance in many compatible plant-virus interactions and its deficiency leads to impairment of the defense responses and increased susceptibility to pathogen attacks $[6,7]$. Compatible interaction is a term broadly used in plant pathology that refers to the interaction between the pathogen and the plant that leads to successful infection, while incompatible interaction stands for successful plant resistance: i.e., the host's ability to limit pathogen multiplication [8]. Recently, we performed a time series analysis of defense responses in compatible potato-PVYNTN interaction using the tolerant cultivar Désirée [4]. Although the plant's fitness was 
almost unaffected, the virus multiplied in the inoculated leaves from five days post inoculation (dpi) on and the spread of viral RNA to upper leaves was detected at $7 \mathrm{dpi}$ [9]. To determine the role of SA in this interaction, the NahG-Désirée transgenic line that expresses salicylate hydroxylase, which catalyzes the conversion of $S A$ to catechol $[7,10,11]$, was also analyzed. In contrast to the non-transgenic plants of cv. Désirée, the SA-deficient transgenic NahG-Désirée showed a greater susceptibility to $\mathrm{PVY}^{\mathrm{NTN}}$. Symptoms in the terms of pronounced yellowing and necrotic lesions started to appear on the site of infection from $4 \mathrm{dpi}$, and became more pronounced in later days. The appearance of the symptoms in NahG-Désirée corresponded to the first detection of viral multiplication at 4 dpi [4]. The dynamics of whole transcriptome changes of cultivar Désirée and NahG-Désirée was analysed in inoculated and systemically infected leaves following $0,1,3,4,5$, and $7 \mathrm{dpi}$.

This paper proposes a methodology aimed at systematic identification of genes that have statistically significant differences of gene expression values between the PVY infected samples and the non-infected (mock) samples at various time points of the recorded time series data. The identified genes present the input for expert analysis and reasoning, aiming to uncover why different potato cultivars differ in terms of resistance to PVY, with the ultimate goal to provide novel insights into the relevant biological processes.

The difficulty of the problem is due to a small number of samples (typically only three samples per a time point per a given potato type) and more than 37,000 candidate genes that have to be tested for their significance. Therefore, we cannot use the standard statistical approaches like the Student's $t$-test or the Mann-Whitney $\mathrm{U}$ test for this dataset. A potentially interesting approach that has been specifically developed for gene expression data performs a differential comparison of sets of genes that are constructed based on their biological functions [12]. A problem of this methodology for our application in the domain of potato plant time series analysis is the often missing information about functions of genes and their functional groups. Standard approaches to longitudinal gene expression analysis are based on spline-based methods for short time sequences [13] and on the approximation of noisy time sequences with simple and smooth functions [14]. These techniques are appropriate for discriminating among complete sequences and are less effective for detecting of differences in specific time points. Finally, techniques for analysis of longitudinal data in medical applications that use within-subject correlation to increase the power of statistical tests [15] are not applicable because potato leaves must be removed from the plants for the analysis of the transcriptome and, therefore, our samples are from physically different leaves.

The novel approach proposed in this work is based on the randomization test concepts [16]. The applicability and usefulness of randomization in gene expression statistical analysis has been previously demonstrated $[17,18]$. In our approach, we construct large stratified randomized gene sets on which we compute the statistical properties of genes, without taking into account the differences between the infected and non-infected plants; we then use this distribution to estimate which of the actual genes have statistically different expression values distinguishing between PVY infected and mock potato samples.

The next section presents the data and the methodology used for the identification of relevant genes from a time series of small data samples. Section 3 presents the results that illustrate the type and quality of the outcomes of this methodology. Finally, Section 4 provides a summary and discusses the limitations of the proposed methodology.

\section{Materials and Methods}

The data analysed in this paper are deposited in the NCBI Gene Expression Omnibus, and are accessible through GSE58593 [19].

\subsection{Data}

Plant material has been grown and manipulated as follows [5]. Potato (Solanum tuberosum L.) cv. Désirée and transgenic potato plants of the same cultivar deficient in SA signaling (NahG-Désirée) 
were propagated in tissue culture. Two weeks after node segmentation, they were transferred to soil in a growth chamber, and kept at $21 \pm 2{ }^{\circ} \mathrm{C}$ in the light and $18 \pm 1{ }^{\circ} \mathrm{C}$ in the dark, at a relative humidity of $75 \% \pm 2 \%$, with $70-90 \mu \mathrm{mol} / \mathrm{m}^{2} / \mathrm{s}^{2}$ radiation (L36W/77 lamp, Osram, Germany) and a 16-h photoperiod. After four weeks of growth in soil, the potato plants were inoculated with PVYNTN (isolate NIB-NTN, AJ585342) using sap prepared from homogenized leaves of tissue culture-grown infected potato plantlets of $\mathrm{cv}$. Pentland squire. For the mock-inoculated plants, the same procedure was performed with sap from healthy potato plants.

On the day of inoculation, three leaves from three non-treated plants for each genotype (Désirée, NahG-Désirée) were collected, which were designated as controls, i.e., at the time point zero days post inoculation (dpi). PVYNTN -and mock-inoculated leaf samples were collected on 1, 3, 4, 5, and 7 dpi. Three plants for each treatment were used.

Total RNA from the inoculated leaves was extracted, DNase treated, purified, and quality controlled as described previously by [5]. A one-colour based hybridization protocol was performed on the custom 60-mer oligo microarrays $(4 \times 44 \mathrm{~K}$; AMADID 015425) designed by the Potato Oligo Chip Initiative [20]. For each sample at least $1 \mu \mathrm{g}$ total RNA was sent for analysis at IMGM Laboratories $\mathrm{GmbH}$, Planegg, Germany. The raw data were analysed in the R Project for Statistical Computing program (R Development Core Team, 2011; version 2.13.2), using the packages Agi4x44PreProcess [21] and Limma [22].

The microarray features were filtered according to the Agilent quality control flags: if the feature was determined to be well above background, if the noise did not exceed a threshold, and if it was not saturated (IsNOTWellAboveBG, IsSaturated, and IsFeatNonUnifOL [20]) in at least $10 \%$ of the total microarray count, then that particular microarray feature was retained for further analysis. The raw data of the remaining 37,865 (from a total of 42,034) features was robust spline normalized ('rsn'; see [23]). The empirical Bayes method [22] was used to detect differentially-expressed genes between PVY ${ }^{N T N}$-and mock-inoculated plants at each time point and for each genotype with corrected $p \leq 0.05$ [24]. Functional analysis of differentially-expressed genes was performed using the MapMan software tool [25] using the ontology adapted for potato [26].

We analysed separately the data from upper non-inoculated and bottom inoculated leaves for the Désirée potatoes, while for NahG potatoes we analysed only the bottom inoculated leaves. In the rest of the paper, Désirée upper leaves are referred to as NT upper, Désirée bottom leaves as NT bottom, while NahG-Désirée potatoes are referred to as NAHG. Table 1 provides an illustration of normalized gene expression data for three out of 37,865 genes. The presented data are for days 1 and 3 for NT upper potato leaves. They illustrate the variability inherent to gene expression measurements.

Table 1. Normalized gene expression data for three genes for NT upper potato leaves. For days 1 and 3 , there are three samples for PVY infected plants and three samples for mock plants. The second row presents the values of the three samples before the start of the experiment.

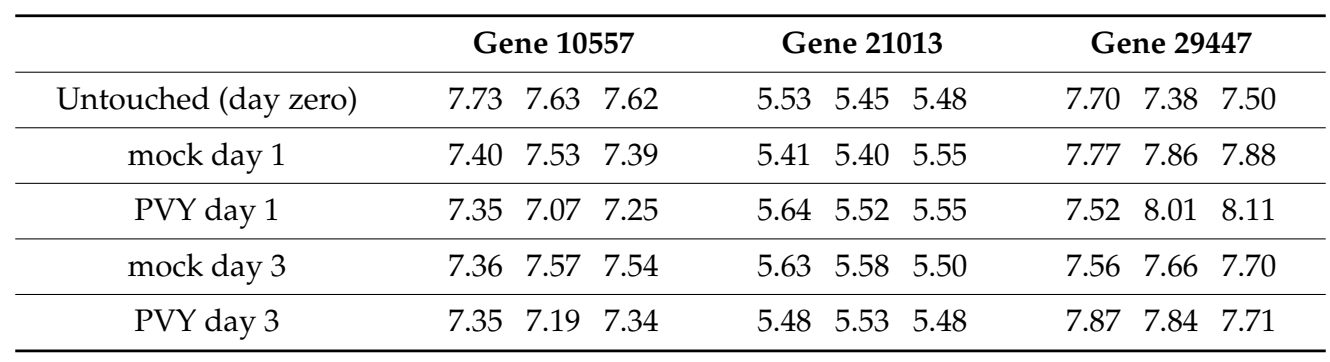

\subsection{Methodology}

For domain expert analysis the most interesting are the genes that significantly change their expression value for infected plants at a specific point in time. Such genes are characterized by two properties: there is statistically significant difference between $P V Y$ values at time points $X$ and $X-1$, while 
the values at these time points do not change in mock samples, and there is a statistically significant difference between PVY and mock values at time point X. Figure 1 illustrates these two conditions.

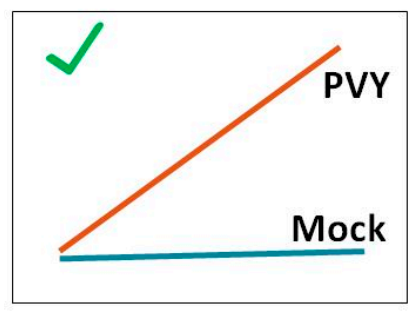

(a)

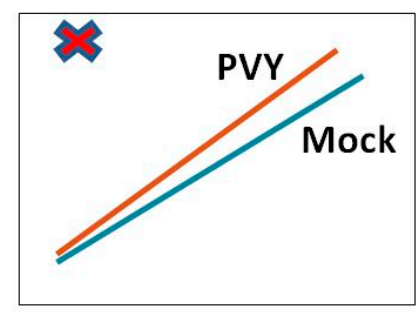

(b)

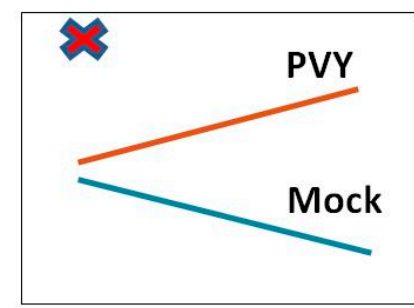

(c)

Figure 1. Illustration of gene expression changes that are interesting for domain expert evaluation in subfigure (a), in contrast to those that are not relevant as shown in subfigures $(\mathbf{b}, \mathbf{c})$.

This means that we have to solve two separate tasks: (1) In the first task, for each time point, we have to identify the genes whose PVY values have changed significantly from the previous time point; and (2) the second task concerns the identification of genes that at the given time points have substantially different values for the PVY and mock samples. Only the genes that satisfy both conditions are interesting for expert analysis. Systematic identification of complete lists of genes that satisfy both conditions is the aim of the proposed methodology.

While the two tasks are performed on different data, the goal of both of them is to identify sets of genes that are substantially differently expressed between the target and the control samples. In the first task the PVY samples at time point $X$ are the target set, while the PVY samples at time point $X-1$ are the control set. On the other hand, in the second task, the PVY and mock samples at time point $X$ are the target and the control set, respectively.

Our basic task is the identification of genes that have statistically significant differences in their expression between the target and the control set. When the target and the control sets are small, in our case consisting of only three samples per each set, the standard statistical tests are not applicable. An alternative approach is possible due to the fact that variance has been stabilized in preprocessing of gene expression data by a model based transformation [23]. The approach consists of two steps: definition of an appropriate measure for computing the difference between the target set and the control set, and by the identification of reliable ranges when the actual values of this measure for some concrete gene can be accepted as statistically significantly different from the no-difference assumption.

A natural selection for the measure of difference between two sets of samples is the relative difference between the average values for samples in different sets, referred as RDA. It is defined as the difference between the average value of the target set and the average value of the control set, divided by the average value for the control set. An alternative measure is relative minimal difference (RMD). In this work we use the latter because of its property that a single measurement error, regardless how large it is, cannot substantially increase its absolute value. This property is important for preventing false positive discoveries. Additionally, for a large fraction of randomly generated data, their RMD values are either equal to zero or they have very small values. This property is, therefore, beneficial for the estimation of distributional characteristics of this measure on random data and the recognition of genes whose measured expression values are significantly different.

\subsubsection{Relative Minimal Distance}

Relative minimal distance (RMD) is defined as follows: its value is positive if all target samples have larger values than the control samples and its value is negative, if all target samples have lower values than the control samples. Furthermore, if there is at least one target sample larger than some control sample and at least one target sample with a value lower than some control sample, then the RMD value is, by definition equal to zero, regardless of the actual values of the samples. RMD also 
always has a value of zero when a pair of target and control samples has identical values. The concept is illustrated in Figure 2.

\begin{tabular}{|c|c|c|c|c|c|c|c|}
\hline$\underset{c 1}{O}$ & $\mathrm{O}_{\mathrm{c} 2}$ & $\mathrm{O}_{\mathrm{c3}}$ & $\mathrm{t}$ & $\begin{array}{l}0 \\
1\end{array}$ & $\begin{array}{l}0 \\
\mathrm{t} 2\end{array}$ & $\mathrm{O}_{\mathrm{t} 3}$ & Positive distance (a) \\
\hline$\underset{\mathrm{t} 1}{\mathrm{O}}$ & $\underset{\mathrm{t} 2}{\mathrm{O}}$ & $\underset{\mathrm{t} 3}{\mathrm{O}}$ & & $\underset{c 1}{\bigcirc}$ & $\underset{c 2}{\mathrm{C}}$ & $\underset{c 3}{\bigcirc}$ & Negative distance (b) \\
\hline$\underset{\mathrm{t} 1}{\mathrm{O}}$ & $\underset{\mathrm{t} 2}{\mathrm{O}}$ & & $\underset{c 1}{\bigcirc}$ & $\underset{\mathrm{t} 3}{\mathrm{O}}$ & $\underset{\mathrm{c2}}{\mathrm{O}}$ & $\underset{c 3}{\mathrm{O}}$ & Distance $=0(c)$ \\
\hline$\underset{c 1}{\mathrm{O}}$ & $\underset{\mathrm{t} 1}{\mathrm{O}}$ & $\underset{\mathrm{t} 2}{\mathrm{O}}$ & & $\underset{\mathrm{t} 3}{\mathrm{O}}$ & $\underset{\mathrm{C} 2}{\mathrm{O}}$ & $\underset{\mathrm{c3}}{\mathrm{O}}$ & Distance $=0(d)$ \\
\hline
\end{tabular}

Figure 2. Illustration of the concept of Relative Minimal Distance between samples t1-t3 in the target set (red) and samples c1-c3 in the control set (white).

When all input target and control gene expression values are positive ( $t x, c x>0)$, non-zero RMD values for positive cases are defined by the relation:

$$
\mathrm{RMD}=(\mathrm{tmin}-\mathrm{cmax}) / \mathrm{cmax}
$$

while for negative cases it is:

$$
\mathrm{RMD}=(\mathrm{tmax}-\mathrm{cmin}) / \mathrm{tmax}
$$

where tmin, tmax, cmin, and cmax are the minimal and maximal values for the samples in the target and control sets, respectively.

An important property of RMD is that for random differences between the target and control sets many RMD values will be equal to zero or their value will tend to be small. For example, in the potato plant gene analysis domain with typically three samples both in the target and the control set we can expect that about $90 \%$ of genes that behave randomly will have RMD values equal to zero. In the case where there were five samples per set available for the analysis, less than $1 \%$ of RMD values for random variables will be different from zero. This property does not depend on the actual intra-set variance. A negative aspect of the RMD measure is that if the intra-set variance is high then it can happen that, even for really significantly different gene sets, the RMD value can also be equal to zero.

Figure 3 presents the distribution of real gene expression data and the corresponding RMD values for one out of 37,865 genes for NAHG potatoes. The RMD values are computed for the differences of gene expression values between the PVY and mock samples. Gene 08407 has a large negative RMD value for day 1 and a small positive RMD vale for day 4. The RMD value for day 1 is statistically significant (see the next section).

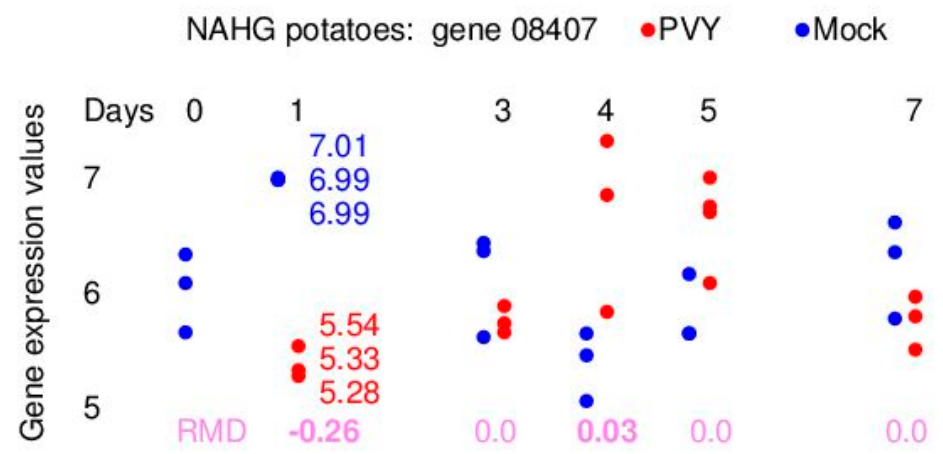

Figure 3. Illustration of changes in time for real gene expression data and the corresponding RMD values. 


\subsubsection{Critical Regions for Accepting RMD Values as Statistically Significant}

Let us assume that we have the target and the control sets with a small number of samples and that there is a large set of genes that are candidates for being uncovered in terms of significantly different values for these two sets. Additionally, let us assume the input data have stabilized variance and there is some measure that can be used to quantify the difference in gene expression values. In the rest of the paper we use the RMD measure defined in the previous section, but note that the methodology can also be used for some other measure of the user's choice. The goal is to identify critical regions of RMD values or, in other words, to compute how large some RMD values must be in order that we can claim that the gene has significantly different expression values for the two sets.

The underlying idea is to construct randomized sets of gene expression values and to compute statistics of RMD values on this data. These statistics will determine the critical regions for RMD values that are acceptable as statistically significantly different from the random data. Since, for randomized data, there are a large number of non-zero RMD values and the probability of positive and negative values is equal, we can conclude that non-zero RMD values computed for randomized data will be normally distributed and that their average value will be equal to zero. This fact is illustrated in Figure 4 for NT-upper potato leaves for day 1. The figure presents distributions of RMD values for real PVY versus mock data and for the randomized data when the PVY and mock values have been shuffled.

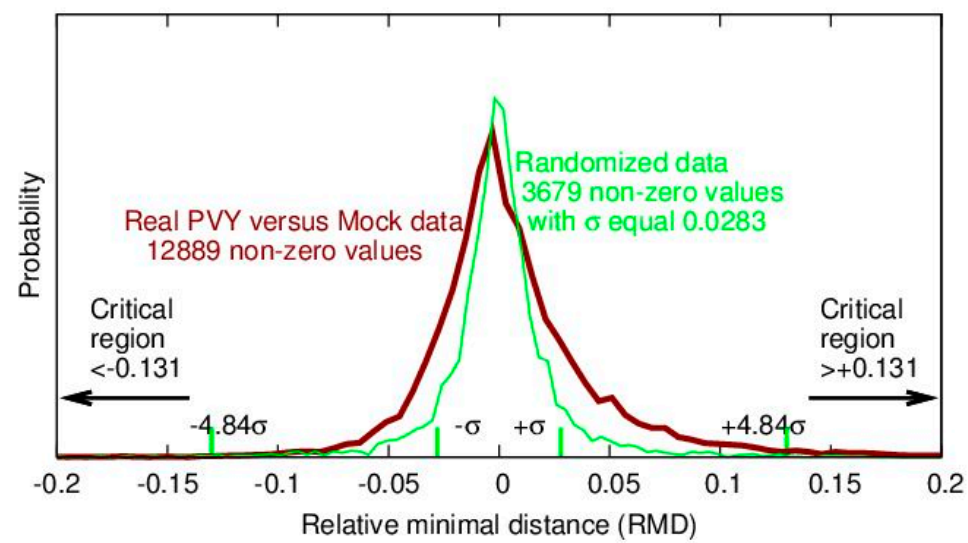

Figure 4. Distribution of real and randomized non-zero RMD values presenting the PVY versus mock data for NT upper potato leaves for day 1.

The critical regions of real RMD values that can be accepted as statistically significant are computed from the standard deviation of the non-zero RMD values for randomized data. The Bonferroni correction has to be used because we have to test the statistical significance of a large number of genes. Theoretically this means that for the two-tailed significance level of 5\% and 37,865 genes that have to be tested, some real RMD value can be accepted as statistically significant if its absolute value is at least 4.84 times greater than the standard deviation computed for non-zero RMD values for randomized data (see Figure 4). Factor 4.84 is determined by using function pnorm from the R language, as follows:

$$
\text { 1-pnorm }\left(4.84=6.5 \times 10^{-7} \sim(0.05 / 2) / 37865\right. \text {. }
$$

Practically the factor can be slightly smaller because for all the genes with real RMD values equal to zero we know that they are not significant. The significance test should be done only for non-zero real RMD values and their number is known for each specific case. Tables 2 and 3 present the data used for computing the critical regions for the first and the second task, respectively. 
Table 2. Computation of regions for statistically significant RMD values for the first task.

\begin{tabular}{|c|c|c|c|c|c|c|}
\hline & $\begin{array}{c}\text { Average RMD Value } \\
\text { for Randomized } \\
\text { Data }\end{array}$ & $\begin{array}{l}\text { Standard Deviation } \\
\text { of RMD Values for } \\
\text { Randomized Data }\end{array}$ & $\begin{array}{c}\text { Number of Non-Zero } \\
\text { RMD Values for } \\
\text { Randomized Data }\end{array}$ & $\begin{array}{c}\text { Number of } \\
\text { Non-Zero RMD } \\
\text { Values for Real Data }\end{array}$ & $\begin{array}{l}\text { Stand. dev. in the } \\
\text { Non-Critical Region (with } \\
\text { Bonferroni Correction) }\end{array}$ & $\begin{array}{c}\text { Absolute Critical Value } \\
\text { for RMD Acceptance as } \\
\text { stat. Significant }\end{array}$ \\
\hline Day & \multicolumn{6}{|c|}{ NT upper } \\
\hline $0-1$ & 0.0000 & 0.0339 & 3603 & 16,936 & $+/-4.68$ & 0.159 \\
\hline $1-3$ & 0.0008 & 0.0305 & 3509 & 10,378 & $+/-4.58$ & 0.140 \\
\hline $3-5$ & -0.0018 & 0.0314 & 3690 & 13,147 & $+/-4.63$ & 0.145 \\
\hline \multirow[t]{2}{*}{$5-7$} & -0.0007 & 0.0321 & 3677 & 5,733 & $+/-4.45$ & 0.143 \\
\hline & \multicolumn{6}{|c|}{ NT bottom } \\
\hline $0-1$ & -0.0001 & 0.0277 & 3850 & 19,444 & $+/-4.71$ & 0.130 \\
\hline $1-3$ & 0.0001 & 0.0285 & 3857 & 8918 & $+/-4.55$ & 0.130 \\
\hline $3-5$ & 0.0001 & 0.0283 & 3833 & 7791 & $+/-4.52$ & 0.128 \\
\hline \multirow[t]{2}{*}{ 5-7 } & -0.0002 & 0.0282 & 3797 & 5450 & $+/-4.44$ & 0.125 \\
\hline & \multicolumn{6}{|c|}{ NAHG } \\
\hline $0-1$ & -0.0002 & 0.0343 & 3658 & 14,028 & $+/-4.64$ & 0.159 \\
\hline $1-3$ & -0.0009 & 0.0346 & 3730 & 16,563 & $+/-4.67$ & 0.162 \\
\hline $3-5$ & 0.0001 & 0.0287 & 2129 & 9119 & $+/-4.55$ & 0.131 \\
\hline $5-7$ & 0.0003 & 0.0259 & 2168 & 4196 & $+/-4.38$ & 0.113 \\
\hline
\end{tabular}


Table 3. Computation of regions for statistically significant RMD values for the second task.

\begin{tabular}{|c|c|c|c|c|c|c|}
\hline & $\begin{array}{c}\text { Average RMD Value } \\
\text { for Randomized } \\
\text { Data }\end{array}$ & $\begin{array}{l}\text { Standard Deviation } \\
\text { of RMD Values for } \\
\text { Randomized Data }\end{array}$ & $\begin{array}{c}\text { Number of Non-Zero } \\
\text { RMD Values for } \\
\text { Randomized Data }\end{array}$ & $\begin{array}{c}\text { Number of } \\
\text { Non-Zero RMD } \\
\text { Values for Real Data }\end{array}$ & $\begin{array}{l}\text { Stand. dev. in the } \\
\text { Non-Critical Region (with } \\
\text { Bonferroni Correction) }\end{array}$ & $\begin{array}{c}\text { Absolute Critical Value } \\
\text { for RMD Acceptance as } \\
\text { stat. Significant }\end{array}$ \\
\hline Day & \multicolumn{6}{|c|}{ NT upper } \\
\hline 1 & 0.0001 & 0.0283 & 3679 & 12,889 & $+/-4.62$ & 0.131 \\
\hline 3 & 0.0001 & 0.0314 & 3587 & 14,459 & $+/-4.65$ & 0.146 \\
\hline 5 & 0.0004 & 0.0247 & 3637 & 8853 & $+/-4.54$ & 0.112 \\
\hline \multirow[t]{2}{*}{7} & -0.0004 & 0.0274 & 3591 & 13,546 & $+/-4.63$ & 0.127 \\
\hline & \multicolumn{6}{|c|}{ NT bottom } \\
\hline 1 & 0.0003 & 0.0254 & 3831 & 8992 & $+/-4.55$ & 0.116 \\
\hline 3 & 0.0004 & 0.0283 & 3831 & 10,454 & $+/-4.58$ & 0.130 \\
\hline 5 & 0.0005 & 0.0373 & 3857 & 10,705 & $+/-4.58$ & 0.171 \\
\hline \multirow[t]{2}{*}{7} & 0.0003 & 0.0397 & 3855 & 8377 & $+/-4.53$ & 0.180 \\
\hline & \multicolumn{6}{|c|}{ NAHG } \\
\hline 1 & 0.0005 & 0.0370 & 3884 & 12,543 & $+/-4.62$ & 0.171 \\
\hline 3 & 0.0001 & 0.0222 & 3790 & 6509 & $+/-4.48$ & 0.097 \\
\hline 5 & -0.0001 & 0.0220 & 2107 & 3896 & $+/-4.37$ & 0.096 \\
\hline 7 & 0.0001 & 0.0361 & 3786 & 4949 & $+/-4.42$ & 0.160 \\
\hline
\end{tabular}


The second, third, and fourth columns of the two tables contain the values computed for randomized data sets among which the standard deviation of non-zero RMD values is the most relevant information. The following two columns contain the number of non-zero RMD values on real data and the corresponding factor for the computation of the critical region with the Bonferroni correction. The last column contains the critical values for each day and the type of potato plants. If the absolute value of RMD for some gene is greater than this value, then the gene can be accepted as having statistically significant differences between the target and the control sets.

An important practical issue is how to generate randomized gene expression data. A simple and effective method is by shuffling the real data:

- The shuffling could be done on the complete gene expression data set irrespective of the meaning of the data. It this case the data are mixed irrespective of the potato types, days, and infection status. This approach can result in incorrect estimation of the standard deviations if the differences between the gene expression data for different potato types and/or PVY versus mock data are large.

- Since we do not have expert knowledge on whether and/or how these differences are relevant, we implemented a better approach based on stratified data shuffling.

- For the first task we shuffle only the PVY values for the same potato type within different days. In this way we randomize time related information and the standard deviation is computed from the real PVY data for the specific potato type as if there were no changes of PVY in time.

- For the second task, we shuffle only PVY and mock data for the specific day and the same potato type. In this way we randomize only the differences between the infected and non-infected plants, while the potential time-related differences and the differences between the potato types remain present also in the randomized datasets.

\subsubsection{Combination of the Two Tasks}

The first step in the identification of significant genes is the computation of critical regions. This is performed by the approach based on the construction of randomized data sets described in the previous section. After we have computed the critical regions, the process of identification of significant genes is very simple: all genes whose absolute value of RMD is above the critical values presented in the last columns of Tables 3 and 4 are accepted as statistically significant. The genes in the resulting lists are ordered according to the descending absolute RMD value.

Table 4. Number of significant genes per task.

\begin{tabular}{|c|c|c|c|}
\hline & $\begin{array}{l}\text { Number of Genes with } \\
\text { Significant PVY Changes } \\
\text { between Two Time Points }\end{array}$ & $\begin{array}{c}\text { Number of Genes with Significant } \\
\text { PVY/mock Differences at the Final } \\
\text { Time Point }\end{array}$ & $\begin{array}{l}\text { Number of Genes } \\
\text { Satisfying Both Conditions } \\
\text { (Final Solution) }\end{array}$ \\
\hline Day & & NT-upper & \\
\hline $0-1$ & 407 & 240 & 180 \\
\hline $1-3$ & 191 & 144 & 41 \\
\hline $3-5$ & 258 & 99 & 7 \\
\hline \multirow[t]{2}{*}{$5-7$} & 40 & 205 & 30 \\
\hline & & NT-bottom & \\
\hline $0-1$ & 943 & 230 & 173 \\
\hline $1-3$ & 96 & 78 & 1 \\
\hline $3-5$ & 89 & 243 & - \\
\hline \multirow[t]{2}{*}{$5-7$} & 30 & 195 & 5 \\
\hline & & NAHG & \\
\hline $0-1$ & 258 & 38 & 1 \\
\hline $1-3$ & 324 & 109 & 35 \\
\hline $3-5$ & 254 & 81 & 35 \\
\hline $5-7$ & 90 & 125 & 4 \\
\hline
\end{tabular}


For each time interval the process has to be repeated two times. First we construct an ordered list of genes that significantly changed their PVY values in the given time interval, followed by computing the list of genes that at the end of the time interval have significantly different values in PVY and mock samples. The final list that presents the result of this methodology consists of the genes that are present in both previous lists and their RMD values in these lists have the same sign.

The result of this process is illustrated in Table 4. The table presents in its second column the number of genes identified as relevant by the first task, in the third column are numbers of relevant genes identified by the second task, while the last column presents the number of genes satisfying both conditions.

The main conclusion from Table 4 is that the final solutions include much less genes than the lists generated by the first and by the second task independently. This means that it makes sense to search for the agreement of both conditions and that the genes in the final solution deserve biological evaluation by the domain expert.

The largest numbers of genes detected as relevant are for the changes in the days 0-1 interval for the NT potatoes, which are large both for the upper and the bottom leaves. The result confirms the expert's knowledge that Désirée reaction to infection is stronger and that the reaction is the strongest immediately after PVY infection.

\section{Results}

The results of the application of the presented methodology are lists of relevant genes that are computed for every time interval. These lists serve as input for expert evaluation. The first step in this evaluation is the analysis of functions of relevant genes. For NT upper leaves functions of three genes whose gene expression values changed most significantly for the first day after infection are: Argnine/serine-rich splicing factor, Thioredoxin, and DNA binding protein. For these three genes their gene expression values of PVY infected samples are statistically significantly higher than the corresponding values of mock samples. For NT bottom leaves, the three genes whose expression values changed most significantly in the same time period are: Chlorophyll $a-b$ binding protein $3 C$ chloroplastic, cell wall protein, and YTH domain family 2. For all these three genes their expression values for PVY infected samples have also increased. In contrast, for NAHG potatoes there is only one gene (Maleylacetoacetate isomerase glutathione S-transferase) whose gene expression values have statistically significantly changed and its expression values in PVY infected samples have changed in the opposite direction.

\subsection{Biological Evaluation of Selected Genes}

In accordance with our previous analysis, photosynthesis-related genes (e.i. gene encoding for chlorophyll $a-b$ binding protein) are differentially expressed in NT bottom leaves at first day post inoculation (dpi). The same day the gene encoding for YTH domain family protein, involved in calcium signalling as well as transcripts for cell wall protein are differentially expressed. SA-deficiency alters fast transcriptional response resulting in maleylacetoacetate isomerase being identified as a relevant transcript. Interestingly, the changes in gene expression in the upper leaves are detected already in the first time period, with transcripts of arginine/serine-rich splicing factor, thioredoxin, and DNA binding protein being differentially expressed, suggesting a fast systemic plant response.

The differences in gene expression of wild type potato plants in the bottom leaves are detected mostly in the last time interval; from 5-7 day transcriptional regulator (MYB transcription factor), gene involved in calcium signalling (calmodulin-like protein) and sugar metabolism (hexulose-6-phosphate isomerase) are regulated upon virus infection. In NahG genotype (NAHG), different transcripts were identified as important regulators showing the importance of SA hormone in the regulatory process. In the upper leaves, reprograming of gene expression is also noted, showing that the plant response is not limited only to the site of virus entry and identifying genes that have a role in systemic plant response (NT upper). 


\subsection{Visualization of RMD Values}

Results visualization is beneficial for understanding of the meaning and for inspecting the relevance of the results. A standard approach is to present the average values and their changes in time. In Figure 5 we present the data for the gene chlorophyll $a-b$ binding protein $3 C$ which has been identified as the most relevant for NT bottom leaves for interval day $0-1$. From the figure it can be concluded that NT upper and NT bottom have higher expression values of PVY infected samples than the values of the mock samples in the period day 1-3. The problem with Figure 5 is that it presents six curves and it is rather difficult to capture all potentially relevant relations.

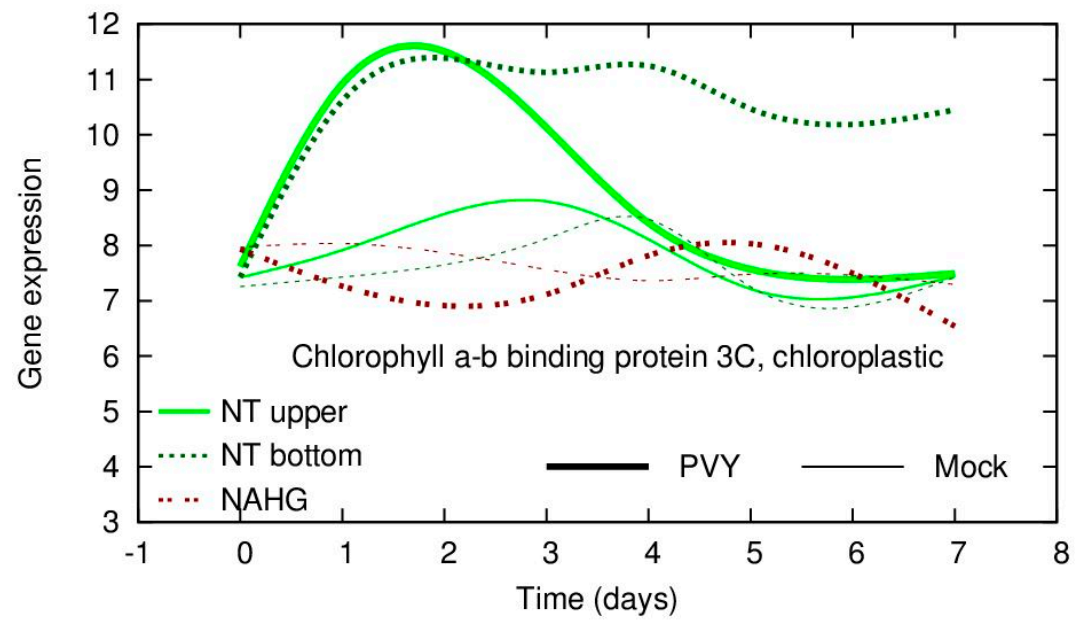

Figure 5. Average values for PVY infected samples (thick lines) and the mock samples (thin lines) for three types of potato leaves for the gene which is detected as relevant for NT bottom leaves in the interval day $0-1$.

A figure that is much easier for interpretation can be obtained by the visualization of RMD values as illustrated in Figure 6.

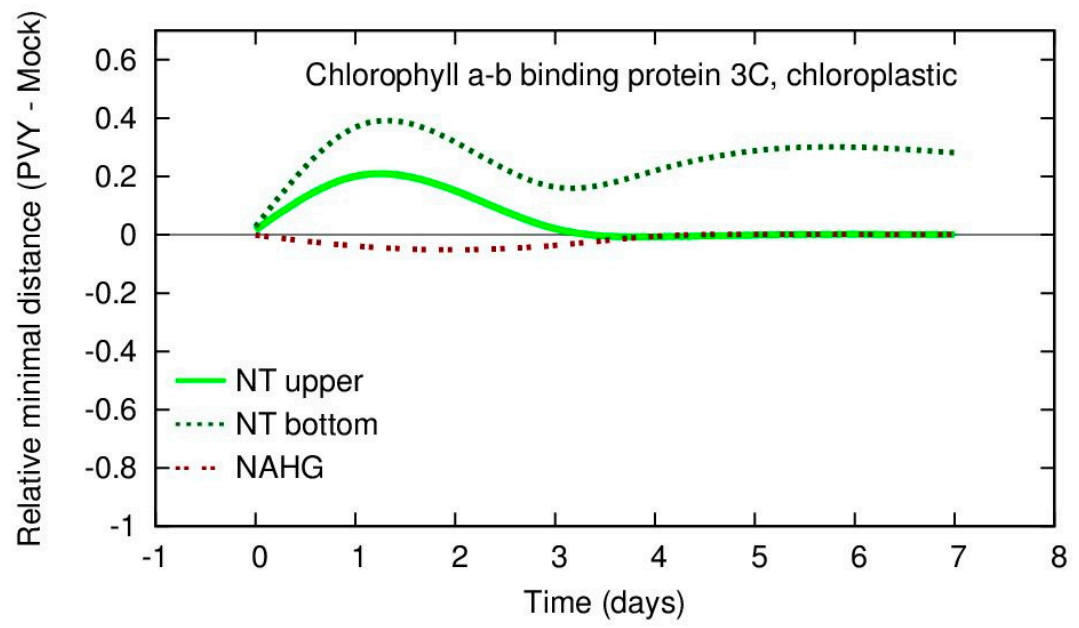

Figure 6. RMD values for three types of potato leaves for the same data as presented in Figure 5.

From Figure 6 it is very clear that the selected gene has a very large difference between the gene expression values of the PVY and mock samples for the NT upper and NT bottom potatoes leaves for days 1-3 and that this value has strongly increased from day 0 . Additionally, the figure demonstrates that in the same period NAGH potato plants have slightly decreased values for this gene. This observation may be a trigger for expert evaluation of the differences between various potato types. By evaluating both Figures 5 and 6 at the same time it may be concluded that for PVY-infected samples, 
the NT upper potato leaves have increased expression values of this gene in the period day 1-3 even more than the NT bottom leaves, but that this difference is not so significant in terms of RMD values. This observation suggests that the variability for PVY and mock data for this gene for the NT upper leaves is large. The result demonstrates the usefulness of visualization of both real gene expression values and their RMD measure for small sets of samples.

\section{Discussion}

High throughput gene expression profiling has emerged over the last decades as one of the most important and powerful approaches in life science research. Additionally, systematic characterization of temporal changes in mRNA levels under different conditions identifies genes relevant for a specific biological response. The task of detecting genes with statistically relevant properties in the setting with a very large number of genes and a small number of samples is a common experimental setting caused by limited sample/tissue access. The contribution of the work is in the definition of a novel measure for characterization of a difference between samples in two classes. When gene expression values have stabilized variance in data preprocessing then stratified data randomization can be used to estimate the statistical properties of this measure for genes that do not differ between target and control samples. Genes whose expression values are statistically significantly differently expressed when compared with control samples and that in some time point have statistically significantly changed expression values in the biological response sequence when compared with the previous time point are selected as specific for this biological response. The approach based on relative minimal distance is very simple and efficient in detecting significant genes with strong stringency. It can be applied to any number of target and control samples, but its application is particularly justified when the number of samples is very small.

The main drawback is that the proposed methodology can result in a very high false negative rate (type II error, a large number of genes that are not detected as significant but that are actually differentially expressed). Namely, a single measurement error may have a consequence that a highly significant gene has a very low minimal distance which can be even equal to zero. This means that the relevance of the uncovered genes in terms of their differential expression is statistically justified, but that we cannot be sure that the resulting set of relevant genes is complete. This fact must be taken into account when analysing the sets of genes detected as relevant.

The methodology enables that the false positive rate (type I error when a gene is detected as significant although it is not significant) can be easily controlled by changing the number of standard deviations in the non-critical region. For example, for the case with the Bonferroni correction for 37,865 genes it is enough to increase the non-critical region from 4.84 to 5.57 standard deviations in order to obtain a probability $p<0.001$ instead of $p<0.05$ that is used in Tables 2 and 3. Some of RMD values in the potato domain are more than ten standard deviations far from the mean values meaning that they are very statistically significant. Six out of seven genes whose functions are presented in Section 3 are statistically significant with $p<0.001$.

Author Contributions: T.S. performed the data curation. D.G. implemented the methodology. K.G. and T.S. performed the validation. D.G. and T.S prepared the original draft. D.M., N.L, and K.G. performed the review and editing.

Funding: This work was financially supported by the Slovenian Research Agency (ARRS) grant HinLife: Analysis of Heterogeneous Information Networks for Knowledge Discovery in Life Sciences (J7-7303) and research programme Knowledge Technologies (P2-0103).

Conflicts of Interest: The funders had no role in the design of the study; in the collection, analyses, or interpretation of data; in the writing of the manuscript, or in the decision to publish the results. 


\section{References}

1. Scholthof, K.-B.G.; Adkins, S.; Czosnek, H.; Palukaitis, P.; Jacquot, E.; Hohn, T.; Hohn, B.; Saunders, K.; Candresse, T.; Ahlquist, P.; et al. Top 10 plant viruses in molecular plant pathology. Mol. Plant Pathol. 2011, 12, 938-954. [CrossRef]

2. Kogovšek, P.; Ravnikar, M. Physiology of the Potato-Potato Virus Y Interaction. In Progress in Botany: Vol. 74; Lüttge, U., Beyschlag, W., Francis, D., Cushman, J., Eds.; Progress in Botany; Springer: Berlin/Heidelberg, Germany, 2013; pp. 101-133. ISBN 978-3-642-30967-0.

3. Singh, R.P.; Valkonen, J.P.T.; Gray, S.M.; Boonham, N.; Jones, R.A.C.; Kerlan, C.; Schubert, J. Discussion paper: The naming of Potato virus Y strains infecting potato. Arch. Virol. 2008, 153, 1-13. [CrossRef]

4. Baebler, Š.; Stare, K.; Kovač, M.; Blejec, A.; Prezelj, N.; Stare, T.; Kogovšek, P.; Pompe-Novak, M.; Rosahl, S.; Ravnikar, M.; et al. Dynamics of Responses in Compatible Potato-Potato virus Y Interaction Are Modulated by Salicylic Acid. PLoS ONE 2011, 6, e29009. [CrossRef]

5. Stare, T.; Ramšak, Ž.; Blejec, A.; Stare, K.; Turnšek, N.; Weckwerth, W.; Wienkoop, S.; Vodnik, D.; Gruden, K. Bimodal dynamics of primary metabolism-related responses in tolerant potato-Potato virus $\mathrm{Y}$ interaction. BMC Genom. 2015, 16, 716. [CrossRef]

6. Jovel, J.; Walker, M.; Sanfaçon, H. Salicylic acid-dependent restriction of Tomato ringspot virus spread in tobacco is accompanied by a hypersensitive response, local RNA silencing, and moderate systemic resistance. Mol. Plant Microbe Interact. 2011, 24, 706-718. [CrossRef]

7. Sánchez, G.; Gerhardt, N.; Siciliano, F.; Vojnov, A.; Malcuit, I.; Marano, M.R. Salicylic acid is involved in the $\mathrm{Nb}$-mediated defense responses to Potato virus $\mathrm{X}$ in Solanum tuberosum. Mol. Plant Microbe Interact. 2010, 23, 394-405. [CrossRef]

8. Glazebrook, J. Contrasting mechanisms of defense against biotrophic and necrotrophic pathogens. Annu. Rev. Phytopathol. 2005, 43, 205-227. [CrossRef]

9. Little, T.J.; Shuker, D.M.; Colegrave, N.; Day, T.; Graham, A.L. The Coevolution of Virulence: Tolerance in Perspective. PLoS Pathog. 2010, 6, e1001006. [CrossRef]

10. Baebler, Š.; Witek, K.; Petek, M.; Stare, K.; Tušek-Žnidarič, M.; Pompe-Novak, M.; Renaut, J.; Szajko, K.; Strzelczyk-Żyta, D.; Marczewski, W.; et al. Salicylic acid is an indispensable component of the Ny-1 resistance-gene-mediated response against Potato virus $\mathrm{Y}$ infection in potato. J. Exp. Bot. 2014, 65, 1095-1109. [CrossRef]

11. Halim, V.A.; Vess, A.; Scheel, D.; Rosahl, S. The role of salicylic acid and jasmonic acid in pathogen defence. Plant Biol. 2006, 8, 307-313. [CrossRef]

12. Hejblum, B.P.; Skinner, J.; Thiébaut, R. Time-Course Gene Set Analysis for Longitudinal Gene Expression Data. PLoS Comput. Biol. 2015, 11, e1004310. [CrossRef]

13. Storey, J.D.; Xiao, W.; Leek, J.T.; Tompkins, R.G.; Davis, R.W. Significance analysis of time course microarray experiments. Proc. Natl. Acad. Sci. USA 2005, 102, 12837-12842. [CrossRef]

14. Berk, M.; Hemingway, C.; Levin, M.; Montana, G. Longitudinal Analysis of Gene Expression Profiles Using Functional Mixed-Effects Models. Adv. Stat. Methods Anal. Large Data-Sets 2013, 57-67.

15. Guo, X.; Qi, H.; Verfaillie, C.M.; Pan, W. Statistical significance analysis of longitudinal gene expression data. Bioinformatics 2003, 19, 1628-1635. [CrossRef]

16. Hooton, J.W. Randomization tests: Statistics for experimenters. Comput. Methods Prog. Biomed. 1991, 35, 43-51. [CrossRef]

17. Kallio, A.; Vuokko, N.; Ojala, M.; Haiminen, N.; Mannila, H. Randomization techniques for assessing the significance of gene periodicity results. BMC Bioinform. 2011, 12, 330. [CrossRef]

18. Wang, X.; Tian, J. A gene selection method for cancer classification. Comput. Math. Methods Med. 2012, 2012, 586246. [CrossRef]

19. GEO Accession Viewer. Available online: https://www.ncbi.nlm.nih.gov/geo/query/acc.cgi?acc=GSE58593 (accessed on 10 January 2019).

20. Kloosterman, B.; De Koeyer, D.; Griffiths, R.; Flinn, B.; Steuernagel, B.; Scholz, U.; Sonnewald, S.; Sonnewald, U.; Bryan, G.J.; Prat, S.; et al. Genes driving potato tuber initiation and growth: Identification based on transcriptional changes using the POCI array. Funct. Integr. Genom. 2008, 8, 329-340. [CrossRef]

21. Lopez-Romero, P. Agi4x44PreProcess. Available online: http://bioconductor.org/packages/Agi4x44PreProcess/ (accessed on 26 October 2018). 
22. Smyth, G.K.; Michaud, J.; Scott, H.S. Use of within-array replicate spots for assessing differential expression in microarray experiments. Bioinformatics 2005, 21, 2067-2075. [CrossRef]

23. Lin, S.M.; Du, P.; Huber, W.; Kibbe, W.A. Model-based variance-stabilizing transformation for Illumina microarray data. Nucleic Acids Res. 2008, 36, e11. [CrossRef]

24. Benjamini, Y.; Hochberg, Y. Controlling the False Discovery Rate: A Practical and Powerful Approach to Multiple Testing. J. R. Stat. Soc. Ser. B (Methodol.) 1995, 57, 289-300. [CrossRef]

25. Thimm, O.; Bläsing, O.; Gibon, Y.; Nagel, A.; Meyer, S.; Krüger, P.; Selbig, J.; Müller, L.A.; Rhee, S.Y.; Stitt, M. MAPMAN: A user-driven tool to display genomics data sets onto diagrams of metabolic pathways and other biological processes. Plant J. 2004, 37, 914-939. [CrossRef]

26. Rotter, A.; Usadel, B.; Baebler, S.; Stitt, M.; Gruden, K. Adaptation of the MapMan ontology to biotic stress responses: Application in solanaceous species. Plant Methods 2007, 3, 10. [CrossRef]

(C) 2019 by the authors. Licensee MDPI, Basel, Switzerland. This article is an open access article distributed under the terms and conditions of the Creative Commons Attribution (CC BY) license (http://creativecommons.org/licenses/by/4.0/). 